- This informal bereavement group work is well attended with positive oral feedback. Formal evaluation underwayresults pending.

\title{
Discussion
}

Benefits Children feel more at ease when handling a pet or when being "carried" by a pony. They can use the animal as distraction from uncomfortable conversations to disclose intimacies/memories that may otherwise be difficult.

Challenges Gaining insurance for the pony component proved challenging but possible. Health and safety issues require stringency.

Conclusion Intrinsic to palliative care and bereavement support is focusing upon the little things that make a person feel respected and loved. We have launched a project that is simple in its theory, but complex and diverse in its positive outcomes.

Recommendations We appeal to all Hospices to explore whether they could use pets in similar ways to support children requiring essential but difficult pre and post bereavement care. Oral presentation will include photos (consented)and interviews from users.

\section{P176 INNOVATIVE PRE AND POST BEREAVEMENT WORK WITH CHILDREN: UNIQUE WAYS WITH ANIMALS}

Jo Fernandes, Alex James The Hospice of St Francis, Berkhamsted, UK

10.1136/bmjspcare-2011-000105.176

Aims Through the catalyst of 'pony experience' bereaved children and parents are facilitated into meaningful conversations with each other and supportive relationships with other families.

Method Once a month bereaved children and families are invited to meet in the Hospice Woodland Walk for Pony Experience, including grooming and riding.

Results

- Commonalty of experience: participants meet other bereaved families, enabling them to share "their stories" should they wish and develop co -supportive relationships.

- Children gain confidence - "Life will go on even without their loved one"

- Observing other children confirms they are not alone, seeing others having fun gives permission for them to do likewise.

- Walking side by side feels less intense, enabling easy conversation and ability to explore and chat at differing levels. 